\title{
SIMPLE HOMOTOPY TYPE OF FINITE 2-COMPLEXES WITH FINITE ABELIAN FUNDAMENTAL GROUP
}

\author{
BY
}

\author{
M. PAUL LATIOLAIS
}

\begin{abstract}
.
THEOREM 1. Let $K$ be a finite 2-dimensional $C W$-complex with $\pi_{1}(K)$ finite and abelian. Then every element of the Whitehead group of $K$ is realizable as the torsion of $a$ self-homotopy equivalence on $K$.
\end{abstract}

THEOREM 2. Homotopy equivalence and simple homotopy equivalence are the same for finite 2-dimensional $C W$-complexes with finite abelian fundamental groups.

0. Introduction. It is known that there exist finite $n$-complexes for all $n>2$ which are homotopy equivalent but not simply homotopy equivalent. In this paper, we show that in dimension 2 homotopy type and simple homotopy type are the same when the fundamental group of the finite 2-dimensional complexes is finite and abelian.

The technique used is to show that all the elements of the Whitehead group of a complex $K$ are realizable as torsions of self-equivalences on $K$.

1. An important example. Dyer and Sieradski [DS] showed in 1973 that two 2-dimensional $\mathrm{CW}$-complexes whose fundamental group was $Z_{n}$ were homotopy equivalent if and only if they were simple homotopy equivalent. The next case to consider would be $Z_{n} \times Z_{m}$. We want to show that any 2-dimensional complex with fundamental group $Z_{n} \times Z_{m}$ realizes all of its Whitehead group as torsions of self-equivalences. ( $\xi(K)$ denotes the group of self-homotopy equivalences of $K$.)

THEOREM 1.1. Let $K$ be the standard 2-complex of the presentation $P=$ $\left\{a, b \mid a^{n}, b^{m},[a, b]\right\}$. Then every element of $\mathrm{Wh}(K)$ is realizable as $\tau(f)$ for some $f \in \xi(K)$.

To prove the above theorem, we need the following three lemmas.

LEMMA 1.2. Suppose $G$ is a group with generating set $\left\{a_{i} \mid i \in I\right\}$ for some index set I. Let $A: Z(G) \rightarrow Z$ be the augmentation map (i.e., the ring homomorphism taking $g$ to 1 for each $g \in G)$. Then each element $\theta \in \operatorname{ker} A$ is of the form

$$
\theta=\sum_{i} \phi_{i}\left(a_{i}-1\right) \quad \text { for some elements } \phi_{i} \in Z(G) .
$$

Proof. See [F, p. 549].

Received by the editors June 1, 1984 and, in revised form, February 18, 1985. Presented to the Society in Louisville, Kentucky, on January 28, 1984.

1980 Mathematics Subject Classification. Primary 57M20, 57Q10.

(C1986 American Mathematical Society $0002-9947 / 86 \$ 1.00+\$ .25$ per page 
LEMMA 1.3. Let $K$ be the standard complex of the presentation $\left\{a, b \mid a^{n}, b^{m}\right.$, $\left.a b a^{-1} b^{-1}\right\}$ and let $\tilde{K}$ be the universal cover of $K$ with a chosen lift $\tilde{e}_{0}$ of the single 0 -cell in $K$. Consider $C_{i}(\tilde{K})$ as a $Z \pi_{1}$-module generated by the preferred lifts of reach $i$-cell. Then $\mathrm{H}_{2}(\tilde{K})$ is generated by the set

$$
\begin{array}{cl}
(a-1) \tilde{R}_{a}, & (b-1) \tilde{R}_{b}, \\
(b-1) \tilde{R}_{a}+\left(\sum_{i=0}^{n-1} a^{i}\right) \tilde{R}_{[a, b]}, & (a-1) \tilde{R}_{b}-\left(\sum_{i=0}^{m-1} b\right) \tilde{R}_{[a, b]} .
\end{array}
$$

Proof. Simple calculations will show that the given chains are cycles and thus represent elements of $H_{2}(\tilde{K})=Z_{2}(\tilde{K})$. To show that they generate all of $H_{2}(\tilde{K})$ (a fact not actually needed in this paper), use a technique similar to Metzler's in [M2, p. 330].

Lemma 1.4. Given a finite 2-dimensional complex $K$ and $Z \pi_{1}$-module map $\phi$ : $C_{2}(\tilde{K}) \rightarrow C_{2}(\tilde{K})$ which commutes with the boundary operator in the sense that $\partial_{2} \phi=\partial_{2}$, then there exists a homotopy equivalence $f: K \rightarrow K$ such that $\tilde{f}_{2}=\phi$ and $\tilde{f}_{1}=$ identity only if the $Z \pi_{1}$-module representation of $\phi$ is invertible.

Proof. This is done by modifying the identity map on $K$ using the Puppe action. This technique is patterned after [DS, p. 41]. For the original reference, see [P].

Suppose $f: K \rightarrow K$ is a homotopy equivalence which induces the identity map on $\pi_{1}(K)$. Let $\tilde{K}$ be the universal cover of $K$. Since $f_{*}: \pi_{1} \rightarrow \pi_{1}$ is the identity, then $f$ is homotopic to a map which induces the identity on $C_{1}(\tilde{K})$. Since we are only interested in homotopy classes of maps, we may assume $f$ is that map. Now $f$ induces the following map on the chain complex of the universe cover $\tilde{K}$ :

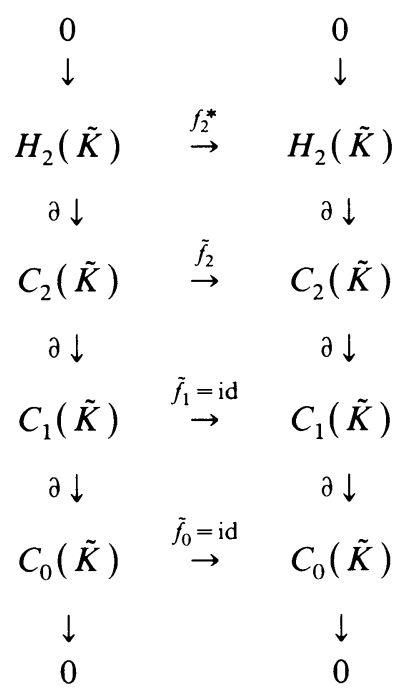

Consider the map id: $K \rightarrow K$. The induced maps on the chain complex of $\tilde{K}$ will all be the identity. Replace $\tilde{f}_{2}$ by $\phi$ in diagram (1). Now we have two chain maps that agree except on $C_{2}(\tilde{K})$. That means that $\phi$-id ${ }_{2}$ will commute in the following 
diagram:

$$
\begin{array}{ccc}
C_{2}(\tilde{K}) & \stackrel{\varphi \text {-id }}{\rightarrow} & C_{2}(\tilde{K}) \\
\partial \downarrow & & \partial \downarrow \\
C_{1}(\tilde{K}) & \stackrel{0}{\rightarrow} & C_{1}(\tilde{K})
\end{array}
$$

Consequently, the image of $\phi$-id will be in $H_{2}(\tilde{K}) \equiv \pi_{2}(K)$. Let $R$ be a 2-cell in $K$ (from the CW-decomposition). Then $\left[\phi\right.$-id] $(\tilde{R}) \in H_{2}(\tilde{K}) \equiv \pi_{2}(K)$. Let $p$ be the representative of the image of $\tilde{R}$ in $\pi_{2}(K)$. Also let $p$ represent the actual map $p$ : $S^{2} \rightarrow K$. Now define $g$ by the composition of the following maps:

$$
K \stackrel{h}{\rightarrow} K \vee S^{2} \stackrel{i \mathrm{~d} \vee p}{\rightarrow} K
$$

where $h$ is the identity on $K \backslash R$ and maps $R$ onto $R \vee S^{2}$ by mapping some $\left(S^{1}, e\right) \subset\left(R, e_{0}\right)$ to $e_{0}$ and the interior disk to $S^{2}$. The rest of $R$ gets "stretched" to cover $R$. Now $\tilde{g}_{2}(\tilde{R})=\phi(\tilde{R})$.

We can modify $g$ using the above technique on the other 2-cells in the decomposition of $K$ to obtain our hypothesized $f$. Using the Five Lemma on diagram (1), $f$ will be a homotopy equivalence if and only if $\phi$ is an isomorphism.

That $\tau(f)=[M]$ comes from direct computation, using Cohen's $\S 15$ and (22.8) in [C].

Proof of Theorem 1.1. Given an element $\phi \in \mathrm{Wh}(K)$, we want to construct a homotopy equivalence $f: K \rightarrow K$, so that $\tau(f)=\phi$. By Lemma 1.4 we merely need to produce an invertible $Z \pi_{1}$-matrix $M$ such that $M$ commutes with the boundary operator and $[M]=\phi \in \mathrm{Wh}(K)$. Since in this case $K$ has three 2-cells, we need $M \in \mathrm{GL}_{3}\left(Z \pi_{1}\right)$.

Let $\bar{a}=a-1$ and $\bar{b}=b-1$. Then by Lemma 1.3 , any matrix of the following form (where the rows represent the images of $\tilde{R}_{a}, \tilde{R}_{b}, \tilde{R}_{[a, b]}$ ) will commute with the boundary operator:

$$
\left[\begin{array}{ccc}
1+\phi_{11} \bar{a}+\psi_{11} \bar{b}, & \phi_{12} \bar{a}+\psi_{12} \bar{b}, & \psi_{11} \sum a^{i}-\phi_{12} \sum b^{i} \\
\phi_{21} \bar{a}+\psi_{21} \bar{b}, & 1+\phi_{22} \bar{a}+\psi_{22} \bar{b}, & \psi_{21} \sum a^{i}-\phi_{12} \sum b^{i} \\
\phi_{31} \bar{a}+\psi_{31} \bar{b}, & \phi_{32} \bar{a}+\psi_{32} \bar{b}, & 1+\psi_{31} \sum a^{i}-\phi_{32} \sum b^{i}
\end{array}\right]
$$

where $\phi_{i j}, \psi_{i j}$ are appropriate elements of $Z\left[\pi_{1}(K)\right]=Z\left[Z_{n} \times Z_{m}\right]$.

Now any element of $\mathrm{Wh}\left(Z\left(Z_{n} \times Z_{m}\right)\right)$ may be represented by a $2 \times 2$-matrix, see Bass [B, p. 183] and Lam [L, p. 143]. Let $\left(\begin{array}{cc}r & s \\ t & u\end{array}\right)$ be that matrix, where $r, s, t$, $u \in Z\left(Z_{n} \times Z_{m}\right)$. Consider the augmentation map $Z\left(Z_{n} \times Z_{m}\right) \rightarrow Z$ which maps the elements of $Z_{n} \times Z_{m}$ to 1 . Call the images of $r, s, t$ and $u$ respectively $r^{\prime}, s^{\prime}, t^{\prime}$ and $u^{\prime} \in Z$. Then we may transform the matrix $\left(\begin{array}{cc}r^{\prime} & s^{\prime} \\ t^{\prime} & u^{\prime}\end{array}\right)$ to $\left(\begin{array}{cc} \pm 1 & 0 \\ 0 & 1\end{array}\right)$ via row and column operators.

We can choose our representative $\left(\begin{array}{ll}r & s \\ t & u\end{array}\right)$ without changing the element in $\mathrm{Wh}\left(Z\left(Z_{n} \times Z_{m}\right)\right)$ in order that $\left(\begin{array}{cc}r^{\prime} & s^{\prime} \\ t^{\prime} & u^{\prime}\end{array}\right)$ transforms to $\left(\begin{array}{cc}+1 & 0 \\ 0 & 1\end{array}\right)$. 
Now use the same row and column operations on $\left(\begin{array}{ll}r & s \\ t & u\end{array}\right)$ that we did on $\left(\begin{array}{cc}t^{\prime} & s^{\prime} \\ t^{\prime} & u^{\prime}\end{array}\right)$. We get a matrix which maps to $\left(\begin{array}{ll}1 & 0 \\ 0 & 1\end{array}\right)$ when $a, b \mapsto 1$. Using Lemma 1.2, this new matrix must be of the form

$$
\left[\begin{array}{ll}
1+\alpha_{1} \bar{a}+\beta_{1} \bar{b}, & \phi_{1} \bar{a}+\psi_{1} \bar{b} \\
\psi_{2} \bar{a}+\beta_{2} \bar{b}, & 1+\phi_{2} \bar{a}+\psi_{2} \bar{b}
\end{array}\right] \text { where } \alpha_{i}, \beta_{1}, \phi_{1}, \psi_{i} \in Z\left(Z_{n} \times Z_{m}\right)
$$

Therefore, we may represent any element of $\mathrm{Wh}\left(Z\left(Z_{n} \times Z_{m}\right)\right)$ in the form (2). If we choose the constants correctly for our matrix (1), and manipulate that matrix to get the form (2), then we will be done. First, letting $\phi_{31}=\psi_{31}=\phi_{32}=\psi_{32}=0$, we get

$$
\left[\begin{array}{ccc}
1+\phi_{11} \bar{a}+\psi_{11} \bar{b}, & \phi_{12} \bar{b}+\psi_{12}(-\bar{a}), & \psi_{11} \sum a+\phi_{12} \sum b^{i} \\
\phi_{21} \bar{a}+\psi_{21} \bar{b}, & 1+\phi_{22} \bar{b}+\psi_{22}(-\bar{a}), & \psi_{21} \sum a+\phi_{22} \sum b^{i} \\
0 & 0 & 1
\end{array}\right]
$$

We then use row 3 to clear column 3 to the equivalent matrix

$$
\left[\begin{array}{ll}
1+\phi_{11} \bar{a}+\psi_{11} \bar{b}, & \phi_{12} \bar{b}+\psi_{12}(-\bar{a}) \\
\phi_{21} \bar{a}+\psi_{21} \bar{b}, & 1+\phi_{22} \bar{b}+\phi_{22}(-\bar{a})
\end{array}\right]
$$

which, with appropriate choice of constants, is equal to the matrix (2). Consequently, we may produce a matrix $M$ which represents any given element of $\mathrm{Wh}(K)$, by Lemma 1.4 , such that $M$ represents $\tilde{f}_{2}$ for some map $f: K \rightarrow K$, with $\tilde{f}_{1}=$ identity. $M$ is invertible since it represents an element of $\mathrm{Wh}(K)$. Therefore, $f$ is a homotopy equivalence. Since $\tilde{f}_{1}$ is the identity map, then $\tau(f)=M$, and therefore all the elements of $\mathrm{Wh}(K)$ are representable.

COROLlaRY 1.5. Let $L$ be a finite 2-dimensional complex with $\pi_{1}(L)=Z_{n} \times Z_{m}$ and $\chi(L)=k$. Then $L$ is simple homotopy equivalent to $K \vee(k-2)\left(S^{2}\right)$, where $K$ is the complex of Theorem 1.1 .

Proof. Since the complex $K$ of Theorem 1.1 realizes all of its Whitehead group as the torsions of self-equivalence on $K$, then by Cohen [C, Theorem 24.4] any complex homotopy equivalent to $K$ is simple homotopy equivalent to $K$. By Dyer [D3], homotopy type of any finite 2-complex with fundamental group $Z_{n} \times Z_{m}$ is determined by the Euler characteristic. Since our example $K$ has minimal Euler characteristic (see Swan [Sw, Proposition 2.1]), the other Euler characteristics may be obtained by wedging $K$ with the appropriate number of copies of $S^{2}$. If $K$ realizes all of its torsion by self-equivalences then so does $K \vee S^{2}$. For if $f: K \rightarrow K$ is a homotpy equivalence, let $f \vee$ id: $K \vee S^{2} \rightarrow K \vee S^{2}$. Then $\tau(f)=\tau(f \vee$ id $)$.

Consequently, given a 2-complex $L$ with $\pi_{1}(L)=Z_{m} \times Z_{n}$ and $\chi(L)=k$, then by Dyer [D3] $L$ is homotopy equivalent to $K \vee(k-2) S^{2}$. But since $K \vee(k-2) S^{2}$ realizes all of its torsion by self-equivalence we know by Cohen [C] that $L$ is simple homotopy equivalent to $K \vee(k-2) S^{2}$. 
COROllary 1.6. Let $K, L$ be finite 2-dimensional $C W$-complexes with fundamental groups $Z_{n} \times Z_{m}$. Then the following are equivalent.

(a) $\chi(K)=\chi(L)$,

(b) $K$ is homotopy equivalent to $L$.

(c) $K$ is simple homotopy equivalent to $L$.

2. The general abelian case. We want to prove that simple homotopy type and homotopy type agree for finite 2-complexes with finite abelian fundamental group. This general situation is unlike the $Z_{n} \times Z_{m}$ case in that homotopy type does not depend only on Euler characteristic. When the Euler characteristic is minimal, homotopy type depends also on bias (see Metzler [M1], Sieradski [S] and Browning [Br]).

According to Browning [Br, Theorem 1.7] and Sieradski [S, Theorem 2], any finite 2-complex of minimal Euler characteristic with finite abelian fundamental group is homotopy equivalent to the standard complex of some twisted (or "untwisted") presentation of the form

$$
\left\{a_{i} \mid a_{i}^{n_{i}},\left[a_{1}^{r}, a_{2}\right],\left[a_{i}, a_{j}\right], i<j, j \neq 2\right\},
$$

where $r<n_{1}$ and $\left(r, n_{1}\right)=1$.

So if we are to use our previous techniques for showing that homotopy type and simple type agree, we not only have to show that the standard complex of the presentation

$$
\left\{a_{i} \mid a_{i}^{n_{i}} ;\left[a_{i}, a_{j}\right] ; i<j ; j=1, \ldots, N\right\}
$$

realizes all of its Whitehead torsion by self-equivalences, we also have to show that the standard complex of any twisted presentation realizes all of its torsion by self-equivalences.

LEMMA 2.1. Let $G$ be a finite abelian group, and let $P=\left\{a_{i} \mid a_{i}^{n_{i}},\left[a_{1}^{r}, a_{2}\right],\left[a_{i}, a_{j}\right] ; i\right.$ $<j ; j \neq 2 ; i, j=1, \ldots, N\}$, where $r<n_{1}$ and $\left(r, n_{1}\right)=1$, be a twisted presentation of $G$. Let $K(P)=K$ be the standard complex of $P$. Then all of the torsion of $\mathrm{Wh}(K)$ is realizable as $\tau(f)$ for some $f \in \xi(K)$.

Proof. The proof proceeds as that of Theorem 1.1. Let $\tilde{K}$ be the universal cover of $K$. Then the following are elements of $\mathrm{H}_{2}(\tilde{K})$ :

$$
\begin{aligned}
& \left(a_{i}-1\right) \tilde{R}_{i}, \quad\left(a_{1}^{r}-1\right) \tilde{R}_{2}-\left(\sum a_{2}^{k}\right) \tilde{R}_{12}, \\
& \left(a_{j}-1\right) \tilde{R}_{i}+\left(\sum a_{i}^{k}\right) \tilde{R}_{i j}, \quad i j \neq 12, i<j, \\
& \left(a_{i}-1\right) \tilde{R}_{j}-\left(\sum a_{j}^{k}\right) \tilde{R}_{i j}, \quad i j \neq 12, i<j .
\end{aligned}
$$

The above restrictions on ij tell us that if we try to use these elements of $H_{2}(\tilde{K})$ to modify the first two rows of the identity matrix, the first column will have no $\left(a_{1}-1\right)=\bar{a}_{1}$ component. So what we will do is ignore the first row and attempt to get our result using the second and third rows. 
Consider the map $\phi: C_{2}(\tilde{K}) \rightarrow C_{2}(\tilde{K})$ which is the identity except:

$$
\begin{aligned}
\tilde{R}_{2} \rightarrow & 1 \cdot \tilde{R}_{2}+C_{11}\left[\left(a_{1}^{r}-1\right) \tilde{R}_{2}-\left(\sum a_{2}^{k}\right) \tilde{R}_{12}\right]+C_{12}\left(a_{2}-1\right) \tilde{R}_{2} \\
& +\sum_{h=3}^{N} C_{1 h}\left[\left(a_{h}-1\right) \tilde{R}_{2}-\left(\sum a_{2}^{k}\right) \tilde{R}_{2 h}\right] \\
& +C_{21}\left[\left(a_{1}-1\right) \tilde{R}_{3}-\left(\sum a_{3}^{k}\right) \tilde{R}_{13}\right] \\
& +C_{22}\left[\left(a_{2}-1\right) \tilde{R}_{3}-\left(\sum a_{3}^{k}\right) \tilde{R}_{23}\right]+C_{23}\left(a_{3}-1\right) \tilde{R}_{3} \\
& +\sum_{h=4}^{N} C_{2 h}\left[\left(a_{b}-1\right) \tilde{R}_{3}+\left(\sum a_{3}^{k}\right) \tilde{R}_{3 h}\right], \\
\tilde{R}_{3} \rightarrow & \cdot \tilde{R}_{3}+C_{31}\left[\left(a_{1}^{r}-1\right) \tilde{R}_{2}-\left(\sum a_{2}^{k}\right) \tilde{R}_{12}\right]+C_{32}\left(a_{2}-1\right) \tilde{R}_{2} \\
& +\sum_{h=3}^{N} C_{3 h}\left[\left(a_{h}-1\right) \tilde{R}_{2}-\left(\sum a_{2}^{k}\right) \tilde{R}_{2 h}\right] \\
& +C_{41}\left[\left(a_{1}-1\right) \tilde{R}_{3}-\left(\sum a_{3}^{k}\right) \tilde{R}_{13}\right] \\
& +C_{42}\left[\left(a_{2}-1\right) \tilde{R}_{3}-\left(\sum a_{3}^{k}\right) \tilde{R}_{23}\right]+C_{43}\left(a_{3}-1\right) \tilde{R}_{3} \\
& +\sum_{h=4}^{N} C_{4 h}\left[\left(a_{h}-1\right) \tilde{R}_{3}+\left(\sum a_{3}^{k}\right) \tilde{R}_{3 h}\right],
\end{aligned}
$$

where the $C$ 's are arbitrary elements of $Z\left[\pi_{1} K\right]$.

If the matrix $M$ representing $\phi$ is invertible, $\phi$ will (by Lemma 1.4) represent the induced map on $C_{2}(\tilde{K})$ of a homotopy equivalence which induces the identity on $C_{1}(\tilde{K})$, since $\partial_{2} \circ M=\partial_{2}$.

$M$ will have the following form:

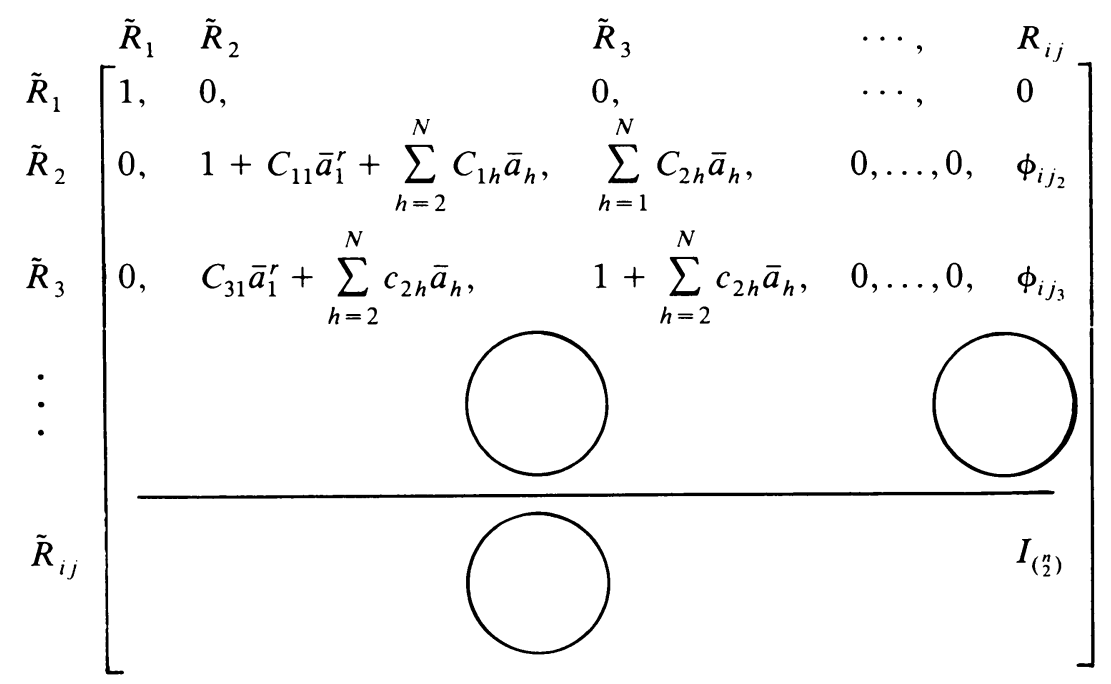


where $\phi_{i j_{g}}(g=1,2)$ is the appropriate row vector, i.e.,

$$
\begin{aligned}
& \phi_{12_{1}}=C_{11}\left(\sum a_{2}^{k}\right), \\
& \phi_{2 h_{1}}=-C_{1 h}\left(\sum a_{3}^{k}\right), \quad 4 \leqslant h \leqslant N, \\
& \phi_{13_{1}}=-C_{21}\left[\sum a_{3}^{k}\right], \\
& \phi_{23_{1}}=-C_{22}\left(\sum a_{3}^{k}\right)-C_{13}\left(\sum a_{2}^{k}\right), \\
& \phi_{3 h_{1}}=C_{2 h}\left(\sum a_{3}^{k}\right), \quad 4 \leqslant h \leqslant N, \\
& \phi_{12_{2}}=-C_{31}\left(\sum a_{2}^{k}\right), \\
& \phi_{2 h_{2}}=-C_{3 h}\left(\sum a_{2}^{k}\right), \quad 4 \leqslant h \leqslant N, \\
& \phi_{13_{2}}=-C_{41}\left(\sum a_{3}^{k}\right), \\
& \phi_{3 h}=C_{4 h}\left(\sum a_{3}^{k}\right) \quad \text { and } \\
& \phi_{23_{2}}=-C_{42}\left(\sum a_{2}^{k}\right)-C_{33}\left(\sum a_{2}^{k}\right), \\
& \phi_{i j_{g}}=0 \quad \text { otherwise. }
\end{aligned}
$$

As before, this matrix is Whitehead equivalent to

$$
\left[\begin{array}{ll}
1+C_{11}\left(a_{1}^{r}-1\right)+\sum_{h=2}^{N} C_{1 h}\left(a_{h}-1\right), & \sum_{h=1}^{N} C_{2 h}(a-1) \\
C_{31}\left(a_{1}^{r}-1\right)+\sum_{h=2}^{N} C_{3 h}\left(a_{h}-1\right), & 1+\sum_{h=1}^{N} C_{4 h}\left(a_{h}-1\right)
\end{array}\right] .
$$

To show that all elements of $\mathrm{Wh}(Z(G))$ can be represented in the above fashion, we invoke Lemma 1.2 using $\left\{a_{1}^{r}, a_{2}, \ldots, a_{N}\right\}$ as the generators of $G$.

Since we only need to consider the above twisted presentations, from Sieradski's Theorem 2 in [2, Lemma 2.1], they give us the following two theorems.

THEOREM 2.2. Let $K$ be a finite 2-dimensional $C W$-complex with finite abelian fundamental group. Then all of the elements of $\mathrm{Wh}(K)$ are realizable as the torsions of self-equivalences on $K$.

THEOREM 2.3. Homotopy equivalence and simple homotopy equivalence are the same for finite 2-dimensional $C W$-complexes with finite abelian fundamental group.

\section{REFERENCES}

[B] H. Bass, Algebraic K-theory, Benjamin, New York, 1968.

[Br] W. Browning, Finite $C W$-complexes of cohomological dimension 2 with finite abelian $\pi_{1}$ (unpublished), Forschungsinstitut fur Mathematik, E.T. H., CH-8092, Zurich, Switzerland.

[C] M. Cohen, $A$ course in simple-homotopy theory, Springer-Verlag, 1970.

[D2] M. Dyer, Simple homotopy types for $(G-m)$-complexes, Proc. Amer. Math. Soc. 81 (1981), $111-115$.

[D3] An application of homological algebra to the homotopy classification of two-dimensional $C W$-complexes, Trans. Amer. Math. Soc. 259 (1980), 505-514.

[DS] M. Dyer and A. Sieradski, Trees of homotopy types of two-dimensional CW-complexes, Comment. Math. Helv. 48 (1973), 31-44. 
[F] R. Fox, Free differential calculus. I, Ann. of Math. (2) 57 (1953), 547-559.

[L] T.-Y. Lam, Induction theorems for Grothendieck groups and Whitehead groups of finite groups, Ann. Sci. Ecole. Norm. Sup. 1 (1968), 91-148.

[M1] W. Metzler, Über den Homotopietyp zweidimensionaler CW-Komplexe und Elementarttransformationen bei Darstellungen von Gruppen durch Erzeugende und definierende Relationen, J. Reine Angew. Math. 285 (1976), 7-23.

[M2] Two-dimensional complexes with torsion values not realizable by self-equivalences, Homological Group Theory, London Math. Soc. Lecture Notes Series 36, Cambridge Univ. Press, 1977, pp. 327-337.

[P] D. Puppe, Homotopiemengen und Ihre Induzieten Abbildungen. I, Math. Z. 69 (1958), 299-344.

[S] A. Sieradski, A semigroup of simple homotopy types, Math. Z. 153 (1977), 134-148.

[Sw] R. Swan, Minimal resolutions for finite groups, Topology 4 (1965), 193-208.

Department of Mathematics, Tulane University, New Orleans, Louisiana 70118

Current address: Department of Mathematics and Computer Science, Dartmouth College, Hanover, New Hampshire 03755 